\title{
Time and foreign exchange markets
}

\author{
Luca Berardi` and Maurizio Serva ${ }^{\dagger}$ \\ * Dipartimento di Ingegneria Elettrica $†$ Dipartimento di Matematica and I.N.F.M. \\ Università degli Studi, L'Aquila \\ Università degli Studi, L'Aquila \\ 67040 Poggio di Roio (AQ) - Italy \\ 67010 Coppito (AQ) - Italy
}

\begin{abstract}
The definition of time is still an open question when one deals with high frequency time series. If time is simply the calendar time, prices can be modeled as continuous random processes and values resulting from transactions or given quotes are discrete samples of this underlying dynamics. On the contrary, if one takes the business time point of view, price dynamics is a discrete random process, and time is simply the ordering according which prices are quoted in the market. In this paper we suggest that the business time approach is perhaps a better way of modeling price dynamics than calendar time. This conclusion comes out from testing probability densities and conditional variances predicted by the two models against the experimental ones. The data set we use contains the DEM/USD exchange quotes provided to us by Olsen \& Associates during a period of one year from January to December 1998. In this period 1,620,843 quotes entries in the EFX system were recorded.
\end{abstract}

PACS numbers: 89.65.Gh; 05.40.Fb

Keywords: Forex markets, time, lags, high-frequency.

Corresponding author:

Maurizio Serva, email: serva@univaq.it, tel. +390862433153, fax. +390862433180 


\section{Introduction}

In the high-frequency arena there are two main-streams about modeling the stochastic properties of quotes. The first approach is to consider quotations as sampled values of an underlying continuous-time random process [6], 7]. Sampling is itself a random operation, thus introducing a twofold uncertainty in the price determination [5], 11]. In this framework, time in the model flows continuously, and is called calendar time.

In the second approach, quoted prices are modeled through a discrete-time stochastic process [12]; in this setting, time is just the natural total order relation among quotations, and it is iso-morphic with the set of non-negative integers (being time 0, the time associated to the first considered quotation). This is the business time approach, and randomness only enters in the determination of prices. It should be pointed out, however, that the waiting times between two quotes are also random quantities, but they are assumed to not contribute to the price determination process.

Whether a calendar-time or a business-time framework should be adopted in modeling the stochastic nature of financial quotes, has been a longly debated issue by the finance research community, and it clearly depends on many factors, like, for example: a) adherence to the physical behavior of reported prices, b) usefulness in terms of a theory to be developed, and c) last but not least, a matter of taste. See, for example [1], 9], [2].

In this paper, we suggest that business time is perhaps a better tool for modeling the asset dynamics than calendar-time. In order to support our claim, we consider: 1) returns corresponding to a given calendar time lag and any business time lag, 2) returns corresponding to the same calendar time lag but having a fixed business time lag. We find out that their statistical properties are different consistently with the business hypothesis and inconsistently with the calendar one. In practice, we estimate some variances and some probability densities whose behavior is different in the two scenarios.

The dataset we use contains the DEM/USD exchange quotes taken from Reuters' EFX pages (the dataset having been supplied by Olsen \& Associates) during a period of one year from January to December 1998. In this period 1,620,843 quotes entries in the EFX system were recorded. The dataset provides a continuously updated sequence of bid and ask exchange quotation pairs from individual institutions whose names and locations are 
also recorded. The reason for using FX data is that this market is not subject to any working time restriction; in fact, it is open 24 hours a day, seven days a week. This is in contrast to stock markets, where artificial time regulation would have made more difficult, if not impossible, to find out the results outlined in this paper.

\section{Business time vs Calendar Time}

\subsection{Calendar Time}

In the calendar time framework, prices are modeled as continuous-time random processes. Clearly, market quotes are not defined for every $t \in \mathbb{R}$, but

only at discrete intervals, whose extensions in time are called calendar lags (usually ranging from 2 sec. to several minutes, sometimes hours). Nevertheless, according to the calendar time picture, prices are usually considered as discrete samples of an underlying continuous-time random process.

The model of price dynamics in calendar time therefore has the following structure:

$$
S(t+\Delta)=S(t) e^{R_{\Delta}(t)}
$$

where $S(t)$ and $S(t+\Delta)$ are the spot prices at times $t$ and $t+\Delta, \Delta$ is an arbitrary calendar time lag and $R_{\Delta}(t)$ is the aggregated return of prices over the time interval $[t, t+\Delta]$.

Considering a framework where prices evolve over the calendar time, it is generally assumed that quotes result from a random sampling at times $t_{0}, \ldots t_{n}$ of the continuous-time underlying process $S(t)$. In a pure calendar time framework such a random sampling is uncorrelated with the process $S(t)$ itself. We observe however that this is only valid as an approximation; indeed, several studies have shown a weak correlation between the sequence of lags and that of returns, among which we cite [10].

The last assumption usually made in order to complete the model description in the calendar time setting is that the variance of $R_{\Delta}(t)$ is a linear function of the calendar time lag $\Delta$, i.e.:

$$
\operatorname{Var}\left[R_{\Delta}(t)\right]=\sigma^{2} \Delta
$$

If the logarithm of $S(t)$ has independent increments the above equation obviously holds and $\sigma$ is the constant volatility. However, it is well known that independence does not hold because of volatility clustering which is due to 
the correlation of the absolute values of returns $[8]$. As a consequence, in spite of a constant volatility, one has a time dependent volatility. Nevertheless, the above behavior of the variance still holds true but $\sigma^{2}$ is now the average of the squared volatility. For our purposes we only assume that the above equality holds and we do not need of specific assumptions concerning volatility behavior.

Let us define the process $M(t)$ as follows:

$$
M(t+\Delta)=M(t)+M_{\Delta}(t)
$$

where $M_{\Delta}(t)$ represents the number of given quotes (samples) in the interval $[t, t+\Delta]$. Clearly, $M(t)$ is a non-decreasing random process assuming integer values. We also observe that $M(t)$ as a function of $t$ is piecewise constant, and its value increases by one each time a quote is given (i.e. at times $\left.t_{0}, \ldots t_{n}\right)$.

Given the assumptions made so far, it follows that the process $M$ and the process $S$ are mutually independent. Hence, it follows that the probability density of returns corresponding to a calendar time lag $\Delta$ is insensitive from the condition that $M_{\Delta}(t)$ is also fixed to a value $m$. In symbols:

$$
P\left[R_{\Delta}(t) \mid M_{\Delta}(t)=m\right]=P\left[R_{\Delta}(t)\right]
$$

and, in particular, the associated variance exhibits the same insensitiveness:

$$
\operatorname{Var}\left[R_{\Delta}(t) \mid M_{\Delta}(t)=m\right]=\operatorname{Var}\left[R_{\Delta}(t)\right]=\sigma^{2} \Delta
$$

Therefore, we can summarize the calendar time hypothesis as follows:

Hypothesis H1: The asset prices evolve over calendar time, i.e. according to the model in Eq. (11) and Eq. (2) holds. Moreover the processes $S$ and $M$ are mutually independent, therefore Eq. (4) and Eq. (5) hold.

Let us anticipate that the main argument of the paper is based on the estimation of the quantities in Eq. (4) and Eq. (5). We will show with enough evidence that the two equalities are largely violated in a way which, on the contrary, is consistent with the business framework.

\subsection{Business Time}

In the business-time approach, price dynamics is modeled as a discrete-time random process. Indeed, the time basis is the ordered sequence of times at 
which prices are quoted in the markets. It is therefore a set isomorphic with the set of non-negative integers. In such a framework the statistic model of price dynamics in the business-time framework is the following:

$$
S(n+m)=e^{R_{m}(n)} S(n)
$$

where $S(n)$ and $S(n+m)$ are the asset price at business times $n$ and $n+m$ while $R_{m}(n)$ is the aggregated return over $m$ consecutive quotes. It is then clear that the only time-dependence affecting the price process is based on the global ordering of events while the return is independent from calendar lag. Notice that we refer to $m$ as the business time lag as opposed to the calendar time lag $\Delta$ introduced in the previous section.

Considering the price dynamics in a business time setting naturally leads to the following assumption:

$$
\operatorname{Var}\left[R_{m}(n)\right]=\hat{\sigma}^{2} m
$$

whose motivation is the same of that provided for the analogous assumption in the calendar time hypothesis. We also define the random process:

$$
T(n+m)=T(n)+T_{m}(n)
$$

where $T(n)$ is the stochastic calendar time at business time $n$ and $T_{m}(n)$ corresponds to the calendar lag $T(n+m)-T(n)$, i.e. the time elapsed from $T(n)$ after the occurrence of $m$ consecutive quotes. It can be readily seen that there is a direct connection between $T(n)$ and the process $M(t)$ defined in the previous subsection. In fact, $M(t)=n$ with $t \in[T(n), T(n+1))$, and, moreover, the following relation holds:

$$
M_{T_{m}(n)}(T(n))=m
$$

for an arbitrary positive integer $m$.

Given the assumption of statistical independence between $S(n)$ and $T(n)$, for a generic $\Delta$ the following relation holds:

$$
P\left[R_{m}(n) \mid T_{m}(n) \in[\Delta-\epsilon, \Delta+\epsilon]\right]=P\left[R_{m}(n)\right]
$$

where $\epsilon$ is a fixed quantity. The above equation states that the probability density of returns corresponding to a business time lag $m$ is insensitive to the condition that the calendar time lag is also fixed to a value around $\Delta$. In particular we have for the variance:

$$
\operatorname{Var}\left[R_{m}(n) \mid T_{m}(n) \in[\Delta-\epsilon, \Delta+\epsilon]\right]=\operatorname{Var}\left[R_{m}(n)\right]=\hat{\sigma}^{2} m
$$


which is the business time analogue of Eq. (5).

Given all the assumptions made so far, we are ready to formulate the hypothesis of prices dynamics in a business time setting.

Hypothesis H2: Asset prices follow the model in Eq. (66) and Eq. (7) holds. Moreover, the processes $S$ and $T$ are independent, it follows that Eq. (8) and Eq. (9) hold.

Before concluding this preliminary outline of the two basic approaches used to describe price dynamics (i.e. calendar time \& business time) we also give another important property of some of the quantities involved so far, which will turn useful in the remaining part of the paper.

With all the positions previously made, let us first observe that the following relation holds: ${ }^{1}$

$$
E\left[M_{\Delta}(T(n))\right]=\alpha \Delta
$$

for a suitable constant $\alpha$. Simply put, this property states that the expected value of the number of quotes in an interval $\Delta$ is proportional to $\Delta$ itself.

Finally, considering the composition of the price process in business time and the process representing the number of quotes in a given calendar time lag $\Delta$, it can be shown that:

$$
\operatorname{Var}\left[R_{M_{\Delta}\left(t_{n}\right)}(n)\right]=\hat{\sigma}^{2} E\left[M_{\Delta}(T(n))\right]=\hat{\sigma}^{2} \alpha \Delta
$$

Thus, in the business time hypothesis, we also expect the variance in (10) to be proportional to $\Delta$.

As already anticipated, all equalities in this subsection are supported by the following statistical analysis confirming the validity the business time framework.

\section{Statistical Estimators}

In this and next section we carry out some experimental analysis in order to best fit the description of prices dynamics choosing between the two distinct possibilities concisely modeled by hypotheses $\mathrm{H} 1$ and $\mathrm{H} 2$.

\footnotetext{
${ }^{1}$ This follows from the stationarity of the process $M_{\Delta}(T(n))$. In particular: $E\left[M_{\Delta}(T(n))\right]$ does not depend on $T(n)$ so we drop the sub case. Moreover, $E\left[M_{k \Delta}\right]=$ $k E\left[M_{\Delta}\right]$, since the average number of quotes in $k$ intervals of the same length sums up to $k$ times the value for the single interval, from which the proportionality follows.
} 
In this section, in particular, we will define some statistical estimators, i.e. functions of the data contained in high frequency time series, and relate them to their probabilistic counterparts defined in the previous section.

Our dataset refers to the FX ratio USD/DM over the whole year 1998 and the price $S_{i}$ we consider in this paper is the half sum of bid and ask (mid-price) while $t_{i}$ denote the time at which the $i$-th price is given. Some automatic filtering procedure is also applied, to remove erroneous recording, which we are able to individuate since they correspond to prices macroscopically different from previous and subsequent ones.

Let $\mathcal{R}=\left\{r_{i}\right\}_{i=0,1, \ldots, L}$ be the series of elementary returns $r_{i}$ defined as:

$$
r_{i}=\log \frac{S_{i+1}}{S_{i}} \quad i=0,1, \ldots, L
$$

and let $\mathcal{T}=\left\{\tau_{i}\right\}_{i=0,1, \ldots, L}$ be the series of temporal lags defined as: $\tau_{i}=$ $t_{i+1}-t_{i}$.

Now consider the series $\mathcal{R}(\Delta, m)=\left\{r_{i}(\Delta, m)\right\}_{i=0,1, \ldots, L(\Delta, m)}$; the $r_{i}(\Delta, m)$ are obtained by summing $m$ consecutive elementary returns (where $m$ is fixed) and subsequently retaining only the $L(\Delta, m)$ sums corresponding to a lag in the interval $[\Delta-\epsilon, \Delta+\epsilon]$ (i.e. the sum of the corresponding $m$ elementary lags $\tau_{i}$ is in the interval $[\Delta-\epsilon, \Delta+\epsilon]$, where $\epsilon$ is also a fixed quantity).

The mean and variance of such a series are respectively defined as:

$$
\begin{aligned}
\mu(\Delta, m) & =\frac{1}{L(\Delta, m)} \sum_{i=1}^{L(\Delta, m)} r_{i}(\Delta, m) \\
v(\Delta, m) & =\frac{1}{L(\Delta, m)} \sum_{i=1}^{L(\Delta, m)}\left[r_{i}(\Delta, m)-\mu(\Delta, m)\right]^{2}
\end{aligned}
$$

We observe that $v(\Delta, m)$ represents an estimation of the quantity $\operatorname{Var}\left[R_{\Delta}(t) \mid M_{\Delta}(t)=\right.$ $m$ ] for the calendar time model; and, as pointed out before, we expect it to be a linear function of $\Delta$, should hypothesis H1 be correct. Moreover, in this hypothesis, we expect this variance to be constant with respect to $m$ if $\Delta$ is fixed.

Alternatively, considering the business time framework, $v(\Delta, m)$ can also be seen an estimator of the quantity $\operatorname{Var}\left[R_{m}(n) \mid T_{m}(n) \in[\Delta-\epsilon, \Delta+\epsilon]\right]$ defined in Eq. (77); should hypothesis $\mathrm{H} 2$ be correct we expect, given $m$, that $v(\Delta, m)$ is approximately constant with respect to $\Delta$. Moreover, in this hypothesis, we expect this variance to be linear in $m$ even if $\Delta$ is fixed. 
With the same set of data $\mathcal{R}(\Delta, m)$ we can can compute the empirical pdf of returns with fixed $\Delta$ and with fixed $m$. This pdf is an estimator of $P\left[R_{\Delta}(t) \mid M_{\Delta}(t)=m\right]$ and also of $P\left[R_{m}(n) \mid T_{m}(n) \in[\Delta-\epsilon, \Delta+\epsilon]\right]$.

Consider now the series $\mathcal{R}(\Delta)=\left\{r_{i}(\Delta)\right\}_{i=0,1, \ldots, L(\Delta)}$ obtained from $\mathcal{R}$ by summing consecutive elementary returns until the corresponding lag becomes equal or greater than $\Delta$. The number of the elements of this series is $L(\Delta)$ and the mean and variance are respectively defined as:

$$
\begin{aligned}
\mu(\Delta) & =\frac{1}{L(\Delta)} \sum_{i=1}^{L(\Delta)} r_{i}(\Delta) \\
v(\Delta) & =\frac{1}{L(\Delta)} \sum_{i=1}^{L(\Delta)}\left[r_{i}(\Delta)-\mu(\Delta)\right]^{2}
\end{aligned}
$$

In the calendar time framework, $v(\Delta)$ estimates the quantity $\operatorname{Var}\left[R_{\Delta}(t)\right]$, defined in Eq. (2). In the business time case, instead, $v(\Delta)$ estimates the quantity $\operatorname{Var}\left[R_{M_{\Delta}\left(t_{n}\right)}(n)\right]$ in Eq. (10). In both cases we expect this quantity to grow linearly with $\Delta$.

With the same set of data $\mathcal{R}(\Delta)$ we can can compute the empirical pdf of returns with fixed $\Delta$ (any $m$ ). This pdf is an estimator of $P\left[R_{\Delta}(t)\right]$.

\section{The choice of the correct model from data anal- ysis}

We have now sufficient information in order to accept or discard hypothesis $\mathrm{H} 1$ and $\mathrm{H} 2$, as a result of an empirical data analysis.

First, we have computed the statistical estimators $v(\Delta)$ and $v(\Delta, m=$ 40) as defined in the previous section and both plotted in Fig. 1 for different values of the calendar time lag $\Delta$. It can be readily seen that while $v(\Delta)$ varies linearly with $\Delta$, the quantity $v(\Delta, m)$ is approximately constant. Indeed, a linear fit was computed in the first case resulting in $v=6.16 E-10 \Delta+8.26 E-8$ and a constant fit in the second resulting in $v=4.83 E-7$.

We recall that, according to the calendar time hypothesis the two lines should be equal and proportional to $\Delta$, while in the business time case the former should be proportional to $\Delta$, while the latter should be constant. The corresponding graphs in fig. 1 seem to suggest that the business time model is more likely valid, while the hypothesis of calendar time dynamics 


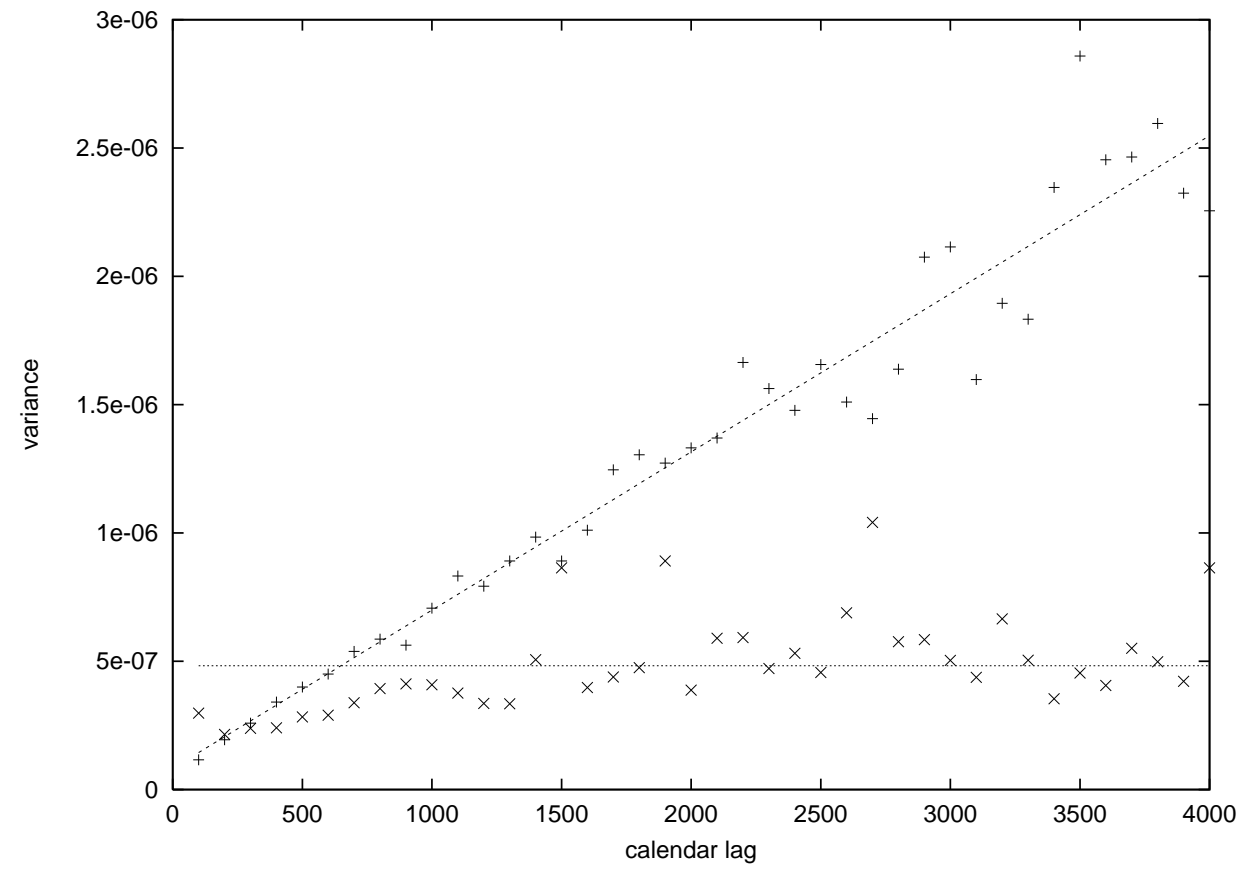

Figure 1: We plot here the statistical estimators $v(\Delta)$ (+ symbols) and $v(\Delta, m)$ with $m=40$ ( $\times$ symbols) for different values of the calendar time lag $\Delta$. It can be readily seen that while $v(\Delta)$ varies linearly with $\Delta$, the quantity $v(\Delta, m)$ is approximately constant. Therefore, if the business time lag is fixed (at $m=40$ ), the variance of the returns does not scale with time lag $\Delta$. This would indicate that business time lag rather than calendar time lag forms the important independent variable. A linear fit was computed in the first case resulting in $v=6.16 E-10 \Delta+8.26 E-8$ and a constant fit in the second resulting in $v=4.83 E-7$. 


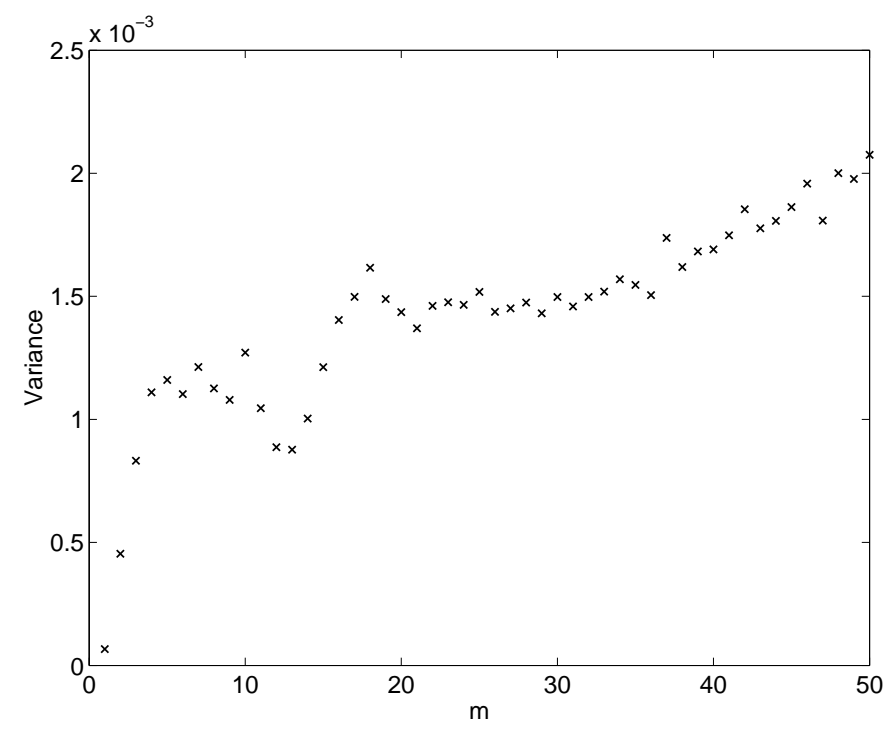

Figure 2: We plot here the statistical estimator $v(\Delta, m)$ with a fixed $\Delta=1000 \pm 50$ for different values of the business time lag $m$. I can be seen that $v(\Delta, m)$ grows with $m$ (even if not linearly in all range considered).

seems to be unlikely. The same kind of behavior can be found if one chooses the value of $m$ in a range between 5 and 100 .

In fig. 2 we also plot the statistical estimator $v(\Delta, m)$ versus $m$ with a fixed $\Delta=1000 \pm 50$. According to the calendar time hypothesis this quantity should be constant while, according to the business time hypothesis, should grow linearly in $m$. The behavior is not linear in all range but, anyway, $v(\Delta, m)$ grows with respect to $m$, which also supports the business time hypothesis. It should be noticed that the choice of other values of the fixed $\Delta$ would not alter this picture.

Second, we consider two distinct series of returns $\mathcal{R}(\Delta, m)$ and $\mathcal{R}(\Delta)$ (respectively $a$ and $b$ ) as defined in the previous section.

Since the minimum lag between two consecutive quotes is equal to 2 seconds in the given database, the two series $a$ and $b$ coincide for $\Delta=2$ sec; formally: $\mathcal{R}(\Delta=2$ sec,$m=1)=\mathcal{R}(\Delta=2 \mathrm{sec})$.

We have subsequently compared the estimated probability density functions (pdf) for the series $a$ and $b$ and the results are shown in fig. 3 ,

The figure is a log-linear plot of different probability densities, For $\Delta=$ $2 s e c$ the pdf the two cases $\mathcal{R}(\Delta=2 s e c)$ and $\mathcal{R}(\Delta=2 s e c, m=1)$ exactly coincide because of the data set characteristics as just explained. For $\Delta=$ 


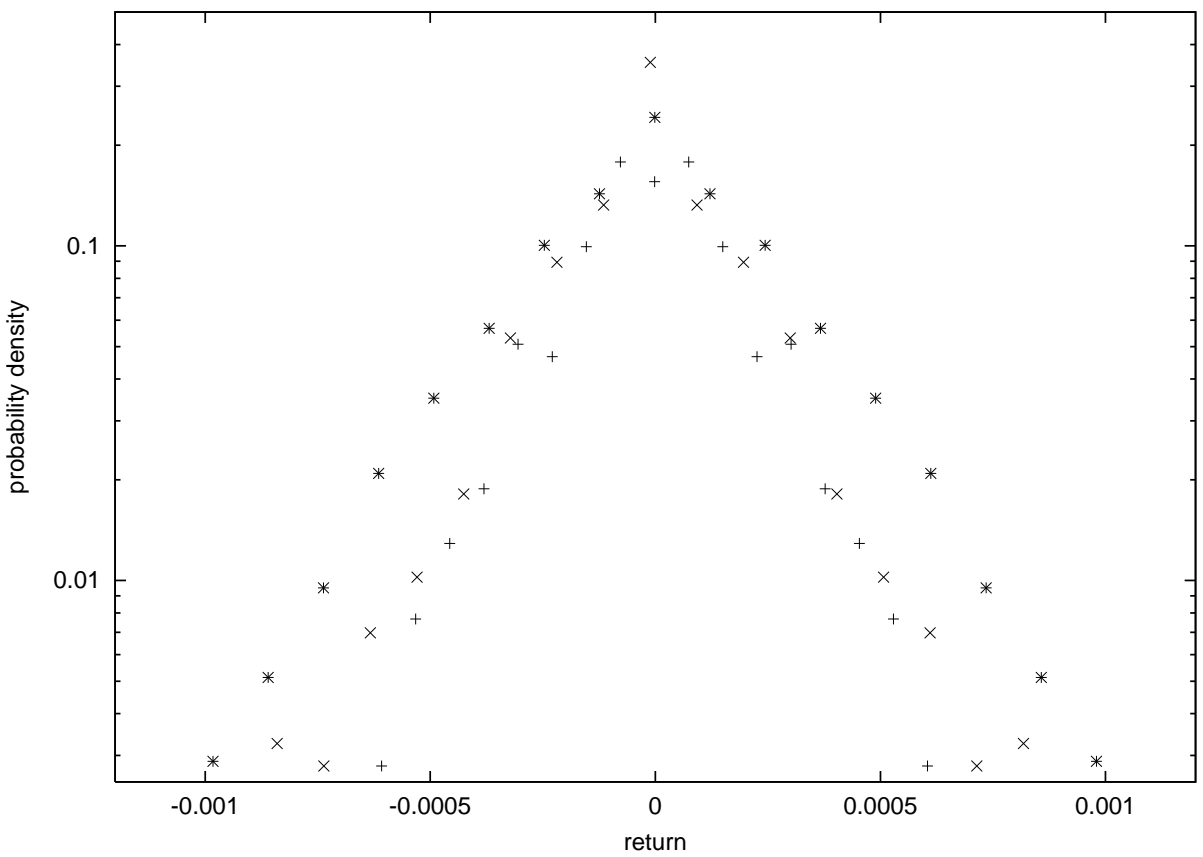

Figure 3: Estimated probability density functions for $\mathcal{R}(\Delta=2 \mathrm{sec})=\mathcal{R}(\Delta=2 \mathrm{sec}, m=$ $1), \mathcal{R}(\Delta=100 \mathrm{sec})$ and $\mathcal{R}(\Delta=100 \mathrm{sec}, m=1)$ in a log-linear plot. The first two pdf (+ symbols) coincide because of the data set characteristics as explained in the text; the pdf for $\mathcal{R}(\Delta=100 \mathrm{sec}, m=1)$ ( $\times$ symbols) is roughly the same of the first two while the pdf for $\mathcal{R}(\Delta=100 \mathrm{sec}$ ) (star symbols) is macroscopically different having larger moments. The significance of the plots lies in the fact that if $m=1$, then a large calendar time seems to make no difference, whereas if $m$ is allowed to vary, then the PDF becomes fat, due to return aggregation. 
100 sec we observe a remarkable difference between the pdf for the series $\mathcal{R}(\Delta=100 \mathrm{sec}, m=1)$ and $\mathcal{R}(\Delta=100 \mathrm{sec})$. The former, in fact, is roughly the same as $\mathcal{R}(\Delta=2 \mathrm{sec})$, while the second is fatter (larger moments).

This fact disagrees with Eq. (4) which is a consequence of calendar time hypothesis. In fact, according to this equation the two pdf corresponding to $\mathcal{R}(\Delta=100 \mathrm{sec}, m=1)$ and $\mathcal{R}(\Delta=100 \mathrm{sec})$ should be equal.

On the contrary, one can immediately see that this result is in accordance with Eq. (8) and, therefore, with business time hypothesis. In fact, $\mathcal{R}(\Delta=$ 100 sec, $m=1)$ and $\mathcal{R}(\Delta=2$ sec, $m=1)$ are roughly the same. This experimental equality simply means that given the value of $m$ returns are substantially insensitive to $\Delta$ as stated in Eq. (8).

In conclusion, this experimental result provides further evidence that the correct model should be the one of the process evolving over business time (hypothesis H2).

\section{Conclusions}

In this paper we suggest that the business time approach is perhaps a better way of modeling price dynamics than calendar time. In order to derive some insight from data we neglect possible autocorrelation between returns and possible autocorrelation between lags assuming implicitly that they would only give a second order correction to our findings. With this simplification our results altogether seem to provide enough evidence for the rejection of hypothesis $\mathrm{H} 1$ (calendar time model) and the acceptance of hypothesis $\mathrm{H} 2$ (business time model). Nevertheless, it should be noticed that hypothesis $\mathrm{H} 1$ assumes that the sampling process is independent of the price evolution. Therefore, our results do not rule out the continuous time model, but rather they show that the the continuous time model would require correlations between processes $M$ and $S$ to in order to fit the data.

The deep reason of the behavior we point out in this paper is that when an asset (at least a forex asset) is not traded, the prices evolution is slow while the evolution is fast when the asset is heavily traded. A faster evolution corresponds to a larger volatility in calendar time [3, 4, therefore, one could even maintain the calendar point of view, but in this case it should accept a seasonal modulation of volatility. The fact that the evolution of a price is slow when there are few transactions is very well known to practitioners but it is still not accepted in its extremal consequence that prices are frozen when 
assets are not traded at all. This is because this behavior is in contrast to the stock market experience where opening prices are different from previous night closing prices. Nevertheless the difference between the two markets is not astonishing if one thinks that the stock market is artificially time regulated, while the forex exchange market is an over the counter (OTC) market not subject to any time restriction.

\section{Acknowledgements}

We would like to thank Filippo Petroni for a number of discussion on the subject and for his advice about the manipulation of the high frequency data sets.

\section{References}

[1] R. Baviera, M. Pasquini, M. Serva, D. Vergni and A. Vulpiani, Correlations and multyaffinity in high frequency financial data sets, Physica A 300, 551-557 (2001).

[2] R. Baviera, M. Pasquini, M. Serva, D. Vergni and A. Vulpiani, Forecast in foreign exchange markets, Eur. Phys. J. B 20 473-479 (2001).

[3] M. Dacorogna, R. Gensay, U. Maller, R. Olsen and O. Pictet, An Introduction to High-Frequency Finance, Academic Press. 2001.

[4] F. Lillo, J.D. Farmer and R. Mantegna, Muster curve for price-impact function, Nature 421, 129-130 (2003)

[5] F. Mainardi, M. Raberto, R. Gorenflo and E. Scalas, Fractional calculus and continuous-time finance II: the waiting-time distribution, Physica A, 287, 468-481 (2000).

[6] R.C. Merton, Continuous-Time Finance, Blackwell Publishers, 1992.

[7] D. Nelson, ARCH Models as Diffusion Approximations, Journal of Econometrics, 45, 7-38 (1990).

[8] M. Pasquini and M. Serva, Multiscaling and clustering of volatility, Physica A, 269, 140-147 (1999).

[9] M. Pasquini and M. Serva, Indeterminacy in foreign exchange markets, Physica A 277, 228-238 (2000). 
[10] M. Raberto, E. Scalas, and F. Mainardi, Waiting-times and returns in high-frequency financial data: an empirical study, Physica A, 314, 751-757 (2002).

[11] E. Scalas, R. Gorenflo and F. Mainardi, Fractional calculus and continuous-time finance, Physica A 284, 376-384 (2000).

[12] S. Taylor, Modeling financial time series, John Wiley \& Sons, New York, (1986). 